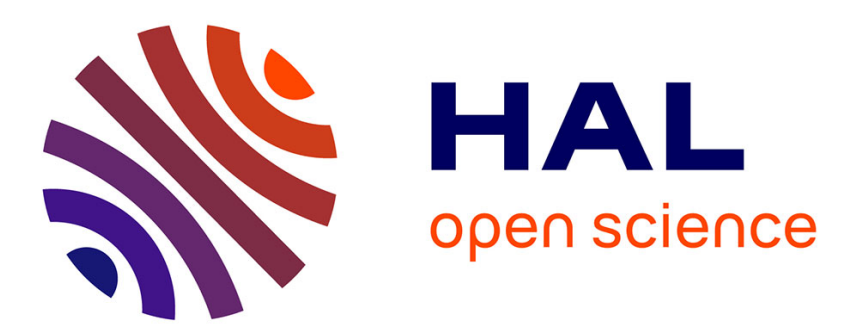

\title{
Characterisation of the EM Disturbances Affecting the Safety of the Railway Communication Systems
}

Virginie Deniau, Nedim Ben Slimen, S. Baranowski, Hamid Ouaddi, Jean Rioult, N. Dubalen

\section{- To cite this version:}

Virginie Deniau, Nedim Ben Slimen, S. Baranowski, Hamid Ouaddi, Jean Rioult, et al.. Characterisation of the EM Disturbances Affecting the Safety of the Railway Communication Systems. European Physical Journal: Applied Physics, 2008, 43 (2), pp.225-230. 10.1051/epjap:2008053 . hal-00505954

\section{HAL Id: hal-00505954 \\ https://hal.science/hal-00505954}

Submitted on 26 Jul 2010

HAL is a multi-disciplinary open access archive for the deposit and dissemination of scientific research documents, whether they are published or not. The documents may come from teaching and research institutions in France or abroad, or from public or private research centers.
L'archive ouverte pluridisciplinaire HAL, est destinée au dépôt et à la diffusion de documents scientifiques de niveau recherche, publiés ou non, émanant des établissements d'enseignement et de recherche français ou étrangers, des laboratoires publics ou privés. 


\title{
Characterisation of the EM Disturbances Affecting the Safety of the Railway Communication Systems
}

\author{
V. Deniau*, N. Ben Slimen*, S. Baranowski**, H. Ouaddi*, J. Rioult* and N. Dubalen*** ${ }^{\dagger}$ \\ *INRETS-LEOST, 20 rue Elisée reclus 59650 Villeneuve d’Ascq, France, virginie.deniau@inrets.fr \\ **IEMN-TELICE, Villeneuve d'Ascq, France, sylvie.baranowski@univ-lille1.fr \\ ***SNCF, IG.LE, 9 Quai de Seine 93584 Saint-Ouen cedex, France, nicole.dubalen@sncf.fr
}

\begin{abstract}
Keywords: Electromagnetic Compatibility, Railway, Communication Systems.
\end{abstract}

\begin{abstract}
To insure interoperability of the transeuropean trains, new transmission systems (GSMR, Eurobalise) are developed in order to permit communications between trains and control centres. These systems have to be available and safe from an electromagnetic point of view, but the normalized methods to characterize electromagnetic noise in railway environment are not convenient for these applications. This paper presents new experimental methods to characterize the electromagnetic noise on board trains which are studied in the framework of the European project "Railcom". The results of measurements realized both in time and in frequency domains, are analysed and some conclusions can be used as a first step to define a new standard for the considered systems.
\end{abstract}

\section{Introduction}

The deployment of the European Railway Traffic Management System (ERTMS) [1] will insure railway interoperability of the transeuropean conventional and high speed trains and then, each of them has to be equipped with adequate telecommunication and locating systems. The management of the European railway traffic will be notably insured by the use of the GSM-R and the Eurobalise telecommunication systems simultaneously.

The Eurobalise system should permit to localise the trains and transmit punctual data from the control centre to the trains. This system requires specific devices localized under the trains allowing both detection and activation of the balises or beacon situated on the ground and the reception of information from the control centre. GSM-R is a communication system which will ensure continuous data and voice transmissions between control centre and trains. For the GSM-R, a specific antenna is fixed on the roof of the train. The reliability of these on board systems can be affected by electromagnetic (EM) disturbances acting on the trains. Thus, it is important to evaluate the EM noise on board, in order to determine the immunity levels that these systems have to verify in this particular environment. The standards applied in order to characterize the EM environment in the railway context are the EN 50121 [2]. These standards notably aim to control the emission levels from the railway infrastructures to the outside world and no method is proposed to characterise the EM environment on board trains. Tests and recommendations found in these standards do not permit to evaluate the EM noise which can be observed at the inputs of the train on-board telecommunication systems. However, EM immunity levels adapted to telecommunication systems used on board trains have to be defined to insure the reliability of these systems in this particular environment.

Previous works [3] were already carried out in order to characterize the EM disturbances produced by the sparks between the pantograph and the catenary and acting on a GSM antenna. These works aimed to observe the disturbances received by a GSM antenna without taking into account the impact on the results of the measurement protocol applied.

The work presented in this present paper aims to propose a standardized method of characterization of the maximum levels of EM noise (over a definite journey) which can affect the GSM-R telecommunication systems on board trains. Consequently, this study analyses the impact of the measurement procedure, configuration and parameters on the results. During a measurement campaign carried out on a moving train, different measurement protocols were tested to characterize the EM environment in order to find the best in term of easiness, reproducibility, and reliability of the results. The studied frequency band is limited to $300 \mathrm{MHz}-1 \mathrm{GHz}$ due to the bandwidth of the available antennas. This paper presents a measurement protocol which can be a first approach in the perspective to develop a new standard adapted to this context.

Firstly, we confront the NF 50121 standards to the current evolution of the railway equipments. Section three describes the measurement configurations applied on board the train and the characteristics of the train, while the two following sections deal with the measurements performed on board the train. Received power where measured in time domain with an oscilloscope and simultaneously in frequency domain with a spectrum analyser; each apparatus was connected to one of two GSM-R antennas fixed on the top of the train. At least, the antenna factor of the GSM-R antennas was studied in order to express the measurements in electric field magnitude and to compare them with standardized limits. 


\section{The Railway EMC standards and the new on- board telecommunication systems}

The standards EN 50121 indicate the methodologies and the limits to apply, relating to the EM emissions and immunity of railway equipments, vehicles and infrastructures. The emissions of the whole railway system, including vehicles and infrastructure are dealt with the section 2 of the EN 50121 [2]. The objective of the tests specified in this standard is to verify that the EM emissions produced by the railway systems do not disturb the neighbouring equipments and systems. The methodology then consists in measuring the radiated EM emissions at a distance of $10 \mathrm{~m}$ from the middle of the tracks and at about $1.5 \mathrm{~m}$ from the ground and to compare them with the maximum allowed levels. The measurements protocol and the limits are specified for the frequencies included between $9 \mathrm{kHz}$ and $1 \mathrm{GHz}$.

The noise levels measured at $10 \mathrm{~m}$ from the tracks do not indicate the EM disturbances that can exist on board the train. However, the knowledge of these on board disturbances is essential to insure that the GSM-R and Eurobalise systems being developed in order to correctly operate in the railway EM environment. In particular, when the EM noise at the regular location of the GSM-R and Eurobalise antennas is known, the immunity of these systems to the railway conditions can be tested in laboratory in reproducing the EM noise observed in situ.

However, to perform EM noise measurement on board trains, the experimental methodology has to be rigorous in order to guarantee representative and reproductive experimental results. Moreover, we have to keep in mind that for the highest frequencies, the presence of specific material (metal, moss of the seat, window glass...) at proximity of the measurement antenna can significantly impact the result. All these aspects have to be taken into account in order to propose a new methodology, which provides results comparable to a unique limit reference.

In a first step, we aimed to propose a methodology to evaluate the EM noise that can act on GSM-R signals transmitted between the trains and the control centre.

\section{Characterisation of the EM noise on the top of the train}

To perform EM noise measurements inside the train, traditional antennas as log-periodic and double ridge antennas could be employed, but above the train, these antennas are not adapted or allowed. On the top of the train, it is necessary to use specific antennas insuring sufficient rigidity, aerodinamism, adequate dimensions and watertightness. Consequently, above the train the measurements were then directly performed with GSM-R antennas which offer all these required characteristics. The models that we employed are double frequency band antennas, covering the frequency band of the GSM-R (870-960 MHz) and the current French railway radio system $(440-470 \mathrm{MHz})$. Their behaviour is then not similar over the $300 \mathrm{MHz}-1 \mathrm{GHz}$ frequency band. These antennas are allowed for GSM-R systems, with a good reception in specific frequency bands. In the case of measurements performed over wide frequency band with GSM-R antennas, it is then necessary to take precautions to obtain relevant experimental results. The measurements presented in this paper were performed on a train including one locomotive and two cars, as shown in Figure 1.

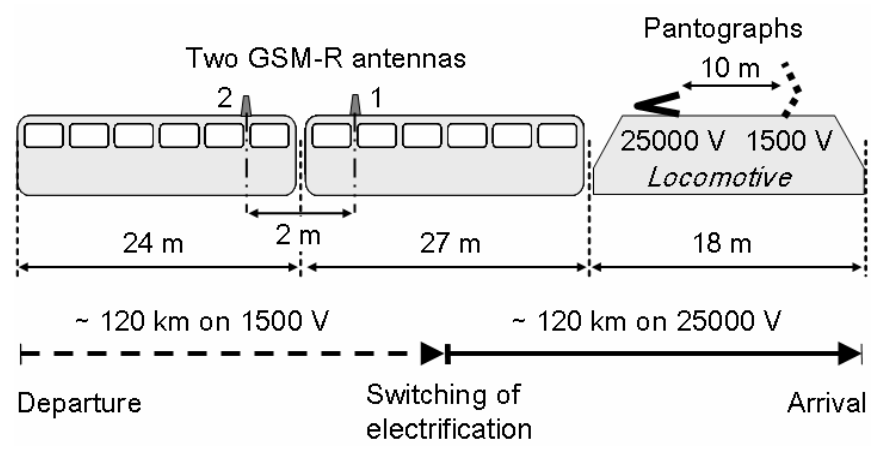

Figure 1: Measurement configuration

The locomotive is equipped with two different pantographs separated by $10 \mathrm{~m}$. The two pantographs allow running on two different supply voltages (1500 V DC and $25000 \mathrm{~V} \mathrm{AC).}$ Two GSM-R antennas were used (called “1” and “2”). One antenna was placed on each car, at $1 \mathrm{~m}$ from the junction between the two coaches. The measurement campaign was performed on a $240 \mathrm{~km}$ run distance. The cruising speed was approximately $120 \mathrm{~km} / \mathrm{h}$ and the duration of the journey was about 2 hours' and 20 minutes.

\section{General about measurements in time and frequency domains}

The characterisation of specific EM environments is generally performed in frequency domain using a spectrum analyser. However, the railway EM environment is notably characterised by brief EM disturbances resulting from the sliding contact between the pantograph and the catenary. The sparks which occur with the poor contact between the catenary and the pantograph produced transient EM emissions. The use of spectrum analyser does not guarantee to totally detect these brief phenomena due to the minimum time necessary to scan the analysis frequency range exceeds the duration on these transient events. Measurements in time domain with an oscilloscope are generally more suitable to characterise transient events.

The main disadvantage of the measurements in time domain is that a signal is recorded each time that a transient event is detected by the oscilloscope. Consequently, at the end of a journey, it can be necessary to analyse an important number of data. Moreover, post processing is required to know the spectrum of the detected disturbances; the Fast Fourier Transform (FFT) has to be applied on all the recorded signals. The frequency domain measurements are currently carried out in combining a peak detection mode and a "Maxhold" function. The "Maxhold" function consists in the comparison of successive recorded spectra and then, to keep the maximum measured value at each frequency. Thus, an unique spectrum gathering the maximum levels obtained at each frequency over a whole journey can be obtained. This 
methodology expedites the analysis of the results. However, the final spectrum collected can depend on the total time duration of measurements and the repetition rate of the sparks. Measurements of transient events such as sparks with spectrum analyser were notably studied in [4].

In our study we performed in parallel time domain and frequency domain measurements on the two GSM-R antennas in order to compare the relevance of the results obtained with the two approaches.

\section{Measurements in time and frequency domains}

The results presented in this abstract were obtained over a 10 minute period at $120 \mathrm{~km} / \mathrm{h}$. The FFT was applied on the events detected by the oscilloscope in order to collect the equivalent spectra. All the spectra were then compared to save only the maximum value corresponding to each frequency over the 10 minutes. Finally, comparisons were performed between the "Maxhold" spectrum measured with the spectrum analyser and the final spectrum obtained from the post processing performed on the time domain data.

Figure 2 gives the results obtained on a part of the $25000 \mathrm{~V}$ AC supply voltage section. These curves represent the maximum powers measured at the antennas output. The black dotted curve which shows a measurement performed with the spectrum analyser when the train moves very slowly, indicates that the noise reference level is $-85 \mathrm{dBm}$.

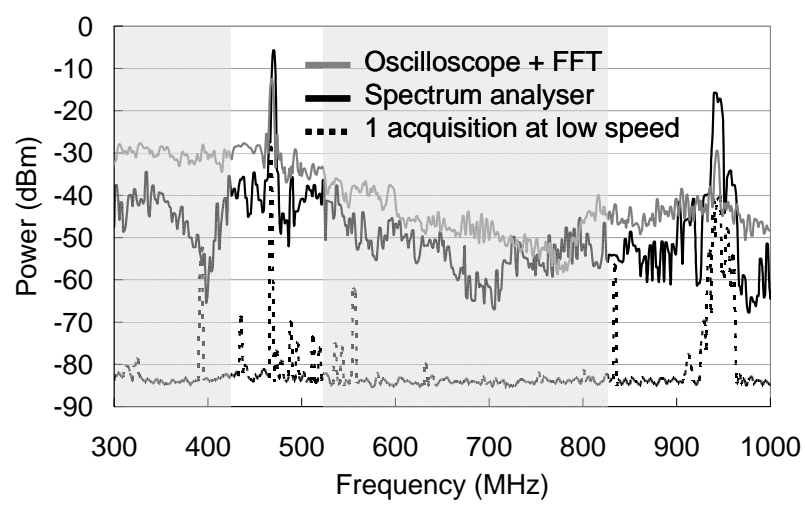

Figure 2: Measurements with spectrum analyser and the FFT of the signals obtained by oscilloscope for the same journey (supply voltage: $25 \mathrm{kV}$ AC)

The difference between this curve and the grey and black curves illustrates the noise specifically produced by the train in regular operating conditions.

In Figure 2, the curves are mainly compared outside the shadow areas which correspond to the bandwidths of the two antennas, i.e. within [420 to 520] $\mathrm{MHz}$ and [820 to 1000] $\mathrm{MHz}$.

Effectively, outside these frequency ranges the reception properties of the antenna are not optimal and do not guarantee the significance of the results.

We notice that the frequency approach slightly underestimate the results obtained with the time domain approach. However, the evolution in frequency of the two results is relatively simular. Consequently, in defining adequate limits levels to each approach, the both time and frequency domain methods could be used. Nevertheless, this requires that the impact of the sparks repetition rate and the measurements duration on the agreement between the time and frequency approaches have to be studied. In our case, during 10 minutes of measurement, 140 transient events were detected by the oscilloscope.

\section{Impact of the antennas locations}

In the experimental results presented, the locations of the antennas were not taken into account. However, according to the origin of the preponderant sources of disturbance, the levels collected by the antennas can vary with their positions. In this section, we have then studied if the measured levels are impacted by the location of the antennas.

Additional measurements were then performed simultaneously on two antennas fixed on the top of the train and significantly spaced in order to verify if the location of the antenna could impact the measured levels.

\subsection{Configuration and protocol measurements}

The two antennas were placed at each extremity of a car and then spaced from $25 \mathrm{~m}$. To have the possibility to fix the antennas at these arbitrary positions, we have to use magnet antennas instead the GSM-R antennas employed in the previous section. The antennas were two identical monopole antennas mainly adapted to the GSM-R frequency band. The frequency range, on which the quality of reception of the antennas is sufficient to collect significant values of the environmental EM noise, is about $600 \mathrm{MHz}-1 \mathrm{GHz}$.

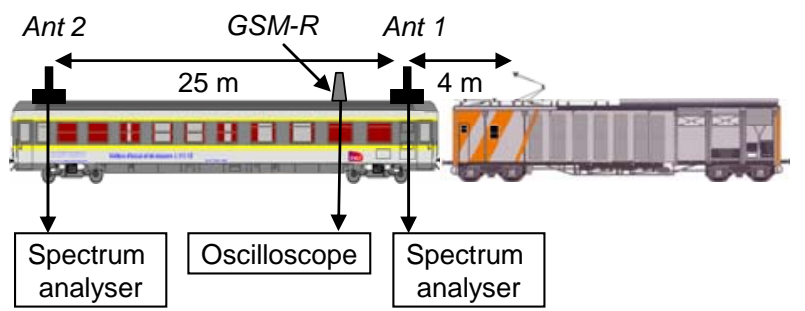

Figure 3: Measurements configuration with two monopole antennas

The measurements were carried out in the frequency domain, using two spectrum analysers connected at the two monopole antennas. The "Maxhold" function was activated over the duration of the measurements. In parallel, knowing that the car was equipped with a GSM-R antenna, we connected it to an oscilloscope in order to count the number of transient events over the measurements time period.

\subsection{Journey characteristics and operating conditions}

These additional measurements were carried on a new TGV line section not totally accomplished, in the proximity of Metz in France on a $25000 \mathrm{~V}$ AC supply voltage section. The measurements were carried out over a trip of about 18 
minutes. The operating conditions of the train were relatively stable during the first 10 minutes while during the 8 last minutes, way-down and up of the pantograph were voluntarily caused.

These measurements were not been able to carry out at the same speed than during the previous measurements campaign presented in section 5 . The speed was about $30 \mathrm{~km} / \mathrm{h}$. However, due to the catenary being newness, about 400 transient events were yet detected by the oscilloscope during the 10 first minutes.

\subsection{Experimental results}

Figure 4 compares the power spectra collected at the two monopole antennas outputs over a 10 minute measurements duration. In this figure, we have also included the result of one acquisition which indicates a $-80 \mathrm{~dB}$ reference noise level.

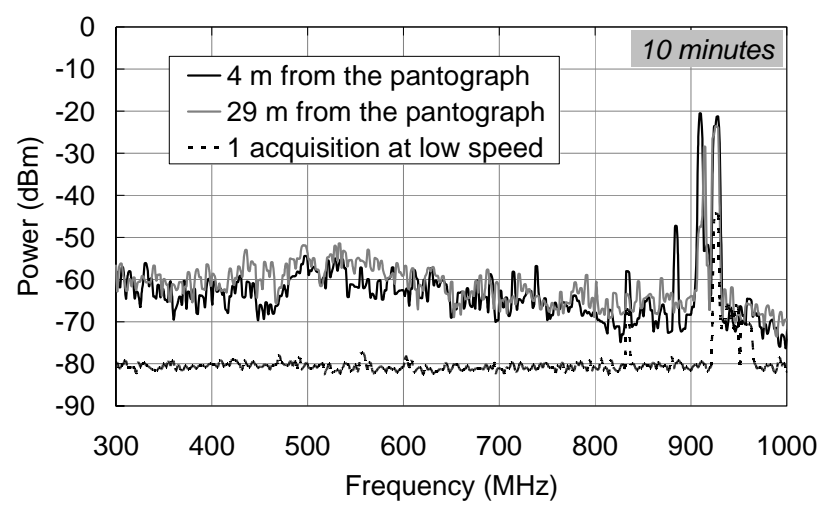

Figure 4: Measurements with spectrum analysers performed simultaneously on two antennas over 10 minutes (supply voltage: $25 \mathrm{kV} \mathrm{AC)}$

We notice that the measured levels are inferior to those presented by the figure 2 . However, we have to keep in mind that the antennas employed were different and the levels measured during the two measurements campaigns can not be directly compared. Moreover, the train speed was different. In figure 4, the spectra measured at the two antennas outputs are nearly similar. Then, in these operating conditions, the location of the antennas do not impact the levels measured. However, if we consider the sparks as punctual emission sources, at the frequency included between $300 \mathrm{MHz}$ and $1 \mathrm{GHz}$, the two antennas were at a sufficient distance from the sources to be in far field. In this case, the radiated emissions locally produced when sparks occur, can have a poor contribution in the disturbance levels measured at the output of the antennas.

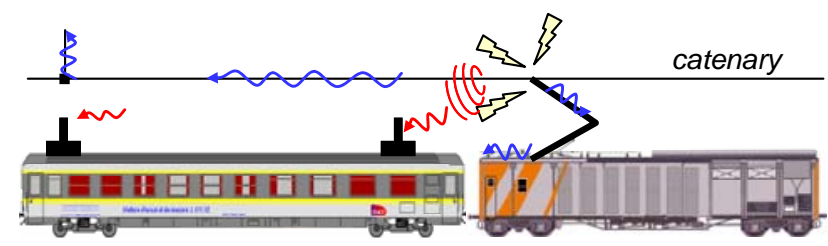

Figure 5: Illustration of the conducted and radiated emissions produced when sparks occur
The disturbances measured by the antennas can mainly be the consequences of the conducted emissions along the different metallic elements as catenary, catenary supports, pantograph, train roof... (Figure 5) and in this case the disturbance source can not be considered as punctual.

Nevertheless, by comparing the results obtained over the total journey including the variable operating conditions (arbitrary up and down of the pantograph) of the 8 last minutes of the journey, some differences appear between the spectra measured at the outputs of the two antennas. The spectra measured over the total duration of the journey are presented Figure 6.

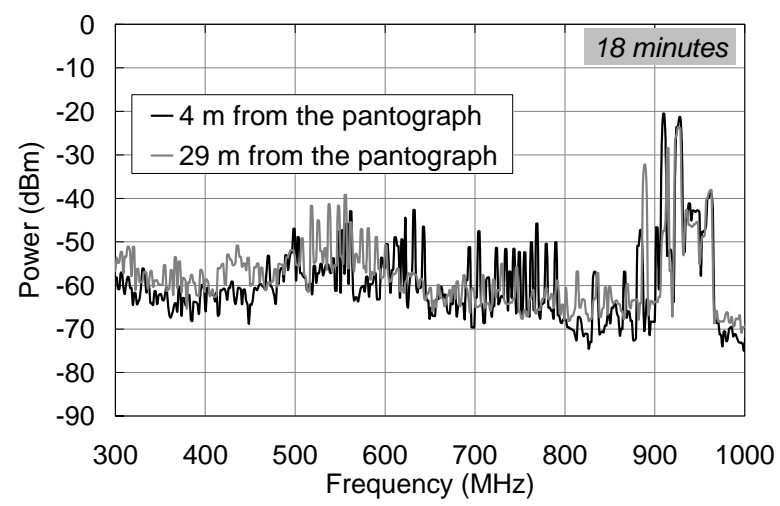

Figure 6: Measurements with spectrum analysers performed simultaneously on two antennas over 18 minutes (supply voltage: $25 \mathrm{kV} \mathrm{AC)}$

On these results, we observed that the spectra measured with the two antennas are not perfectly identical. However, we do not notice a superior magnitude measured by the antenna the nearest from the pantograph. Consequently, these different results seem to indicate that the sparks can not be considered as punctual disturbances sources and we can not establish direct relations between the distance pantograph - antenna and the measured levels.

\section{Comparison with standardized emission limits}

The EN 50121 standards specify the maximum magnitude $\mathbf{E}_{10 \mathrm{~m}}$ of the electric (E) field measured at $10 \mathrm{~m}$ from the middle of tracks. However, in the case that measurements were carried out at a lower distance $\mathbf{D}$, these standards indicate Equation (1) to calculate the electric field levels $\mathbf{E}_{\mathbf{D}}$ to not exceed.

$$
E_{D}=E_{10 m}-20 \cdot \log (D / 10) .
$$

This relation (1) is given to be available between $110 \mathrm{MHz}$ and $1 \mathrm{GHz}$. However, the use of (1) is inadequate when the distance D is small because the EN 50121 advises against measurements at distance inferior to $3 \mathrm{~m}$ due to the body effect of the train on the results. Moreover, if we employ this equation to calculate the limit of $E$ field at the location of the GSM-R antenna which is at the centre of the tracks, the limit tends toward infinite. Initially, this relation is then not 
destined to provide limits of E field which can be compared to E field levels measured on board trains. Consequently, the comparisons which are carried out in this section with the standardized limits only aim to confront the results obtained by our measurements protocol with quantitative references.

Thus, from the results obtained during the measurement campaign presented in the section 5, the E field levels existing on the top of the train were deduced in order to quantify the difference with the limits of E field specified in the EN 50121. We had then previously characterized the "antenna factor" between $300 \mathrm{MHz}$ and $1 \mathrm{GHz}$ of the GSM-R antennas in order to translate the power measured at the antennas outputs in an equivalent environmental $\mathrm{E}$ field $(\mathrm{dB} \mu \mathrm{V} / \mathrm{m})$.

Figure 7 gives the E field measured on the top of the train in applying the antenna factor of a GSM-R antenna to the powers measured by the time domain method.

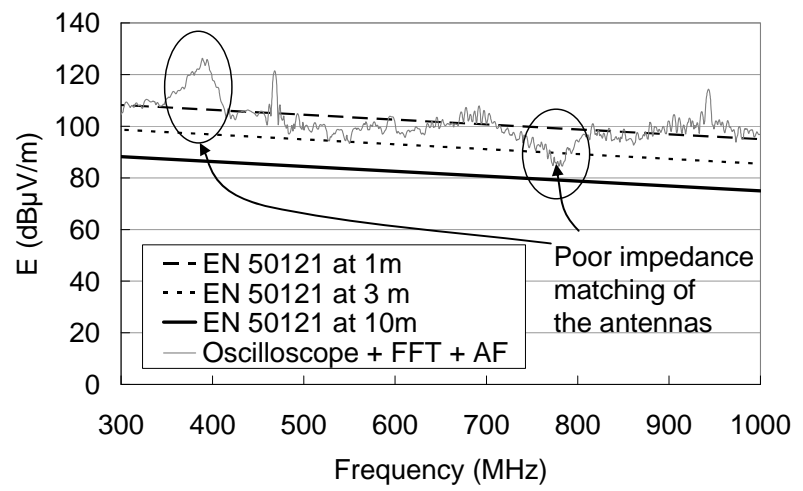

Figure 7: Comparison between the Electric field measured on board and the maximum limits indicated in the EN 50121 at difference distances.

This curve presents two significant variations at about $400 \mathrm{MHz}$ and $780 \mathrm{MHz}$. At these frequencies, the GSM-R antenna has very poor impedance matching and its antenna factor can not be significant. Consequently, at these frequencies, the results can not be taken into account and these variations can not be attributed to E field variations.

In order to confront the field levels with quantitative reference, the maximum limit at $10 \mathrm{~m}$ from the tracks specified in the EN 50121 is given by the black line. Obviously, the results exceed the limit at $10 \mathrm{~m}$ due to the measurements being performed on the top of the train. We have also represented by the two dashed lines the $\mathrm{E}$ field limits calculated with (1) for measurements performed at $1 \mathrm{~m}$ and $3 \mathrm{~m}$.

We note that the E field measured levels are approximately included between the limits calculated for $1 \mathrm{~m}$ and $3 \mathrm{~m}$ but this is only a quantitative comparison and actually none rational explanation or conclusion can be made.

Effectively, we have to keep in mind that during the measurements performed on board, the antenna was necessary fixed on the roof of the train. The body of the vehicle therefore constituted a large ground plane and probably impacted the results. Consequently, above the vehicle the free space conditions are not verified and the equation (1) is not adapted to the field propagation conditions. Thus, the characterisation of the EM environment on board the train requires adequate methodology and specific reference levels. This observation only shows that the E field levels deduced from the powers received by the GSM-R antennas and their antenna factors are coherent whereas these antennas are not specifically designed for electromagnetic environment characterisations.

\section{Conclusions}

In order to propose an adequate methodology to evaluate the EM noise which can affect on-board telecommunication systems, two approaches were tested for electromagnetic noise measurements on moving trains. The time domain measurements method is well appropriate to collect the whole spectrum of time transient emissions, but requires post processing. Consequently, measurements in frequency domain being easier to carry out are suitable for standardization. Nevertheless, the frequency domain approach can slightly underestimate EM noise levels and the agreement with the results given by the time domain approach can depend on the duration of the measurements and the occurrence of the sparks.

During this measurement campaign in situ, representative data of the railway EM environment were collected using particular measurement methodologies. However, the main objective of this study will consist of producing railway noise shape in laboratory in order to test new systems or prototypes for example. The frequency domain measurement approach will then be accurately calibrated in relation to time domain measurements. The impact of the transient events repetition rate will notably be studied and methodologies for immunity testing adapted to the on-board equipments and systems will be proposed.

\section{Acknowledgements}

This work was carried out within the framework of the RAILCOM project and benefited of the financial support of the PCRD 6. The measurement campaigns would not been able to be carried out without the contribution of the SNCF.

\section{References}

[1] http://www.ertms.com/.

[2] European Standards EN 50121:2006, Railway applications-Electromagnetic compatibility.

[3] M. Szelag, M. Klingler and Consortium Morane, "Statistical Characterisation of Electromagnetic Disturbances Produced by the Sparks between Pantograph and Catenary of TGV on a GSM antenna, International Symposium on EMC, September 1998, Rome, Italy.

[4] A. Wisten., P. Makikaltio, "Methods Of Measuring Electromagnetic Emission from Trains," EMC Europe 2004, Eindhoven, The Netherlands. 